\title{
Critical Analysis of Partial Discharge Dynamics in Air Filled Spherical Voids
}

\author{
G. Callender ${ }^{1}$, I. O. Golosnoy ${ }^{1}$, P. Rapisarda ${ }^{2}$ and P. L. Lewin ${ }^{1}$ \\ The Tony Davies High Voltage Laboratory ${ }^{1}$ \\ Vision, Learning and Control Group ${ }^{2}$ \\ School of Electronics and Computer Science \\ University of Southampton \\ Southampton, SO17 1BJ, UK \\ Email (1): gc1g13@soton.ac.uk
}

\begin{abstract}
In this paper partial discharge (PD) is investigated inside a spherical air filled void at atmospheric pressure using a drift diffusion model. Discharge dynamics consisted of an electron avalanche transitioning into positive streamer, in agreement with earlier work on dielectric barrier discharges. Different model configurations were utilised to test many of the concepts employed in semi-analytical PD activity models, which use simplistic descriptions of the discharge dynamics. The results showed that many of these concepts may be erroneous, with significant discrepancies between the canonical reasoning and the simulation results. For example, the residual electric field, the electric field after a discharge, is significantly lower than the estimates used by classical PD activity models in the literature.
\end{abstract}

\section{Introduction}

The term partial discharge (PD) is used to describe the localised breakdown within high voltage insulation that does not fully bridge the gap between electrodes, for example discharges occurring within solid insulation systems with gaseous voids [1]. In some cases PD activity can significantly enhance defect severity, leading to premature failure of equipment. As such PD measurements are a widely used technique to determine the health of insulation material within high voltage plant. A significant amount of research activity has focused on improving the measurement, detection and source recognition [2]. Models of PD have also received research attention as they improve our physical understanding and in some cases allow conclusions to be drawn on conditions within defects [3]. The foundational work in PD modelling began with the work of Niemeyer in the 1990's 3 5. Defects within solid insulation were described as equivalent ellipsoids and the electric fields calculated using appropriate geometry parameters. The simulation of PD activity has primarily focused on spherical or cylindrical air-filled voids surrounded by a dielectric material between parallel plates, and the following discussion will also be restricted to these cases.

More recently finite element models have been developed that allow the electric field to be determined throughout the PD system [6, 7]. Models that consider the plasma dynamics for many PD systems of interest have received relatively little research attention. It has been determined that for a single PD occurring between dielectric barriers the discharge mechanism consisted of an electron avalanche transitioning into a positive streamer [8]. A one dimensional model for single discharges in air gaps has also been shown to give good agreement with experiment [9]. Plasma models that consider multiple PDs have also been considered [10, 11]. In the case of [10] the first order scheme used, by necessity to reduce the computational cost, could lead to high numerical diffusion which means sharp gradients may not be resolved accurately. Very recently a model of multiple PDs in a spherical air filled void has also been presented. In other work, [11, a small number of PDs were simulated in a spherical void. Although not part of PD research explicitly, discharges within spherical bubbles in dielectric liquids are physically similar system, i.e. discharges within a gaseous sphere within a homogeneous dielectric material, which have been investigated using simulation [12, 13]. 
Simulation work has shown that when the physical conditions are similar to that of a PD system, these discharges consisted of an electron avalanche transitioning into a positive streamer [14, in agreement with the work on dielectric barrier discharges. The plasma dynamics are distinct due to the containment of the discharge within the void limiting the spread of the plasma. In the research of discharges within bubbles, the emphasis has been on the initiation of the discharge and the plasma dynamics.

A model that considers plasma dynamics for a large number of PDs is currently computationally prohibitive, and as such models that consider a highly simplified description of discharges are still often used for electrical engineering applications [6, 7. Recent finite element models of PD activity typically consider a discharge as a process which alters the surface charge density on the surface of the void in which it occurs [6. Surface charge density distributions are assumed to be bipolar, and surface charge from PDs is deposited 'on top' of charge on the void surface from previous discharges. PDs can only occur when the electric field in the void is sufficiently high, and deposit surface charge such that the electric field is reduced to a residual value. In order to test whether this simplified description is valid, it is necessary to use a model that can provide an accurate model of the plasma dynamics for a PD system. The aim of this work is to assess some of the critical assumptions made in these models by implementing a simple drift diffusion model for three charged species. The intention of this work is distinct from the work on discharges within bubbles. The focus here is on the calculating current pulses and charge magnitudes across the ground electrode and the surface charge density distributions on the void surface from discharges, which are quantities of interest from the perspective of electrical engineering.

The PD system considered in this work is a spherical void inside epoxy resin between parallel plate electrodes. The motivation for this choice is twofold. Firstly, it has been considered by previous researchers in the literature [3, 6, 15, and secondly it is indicative of a situations that can occur in epoxy resins in high voltage plant due to errors in the curing process. To narrow the scope an air filled void at atmospheric pressure will be considered. This is designed to be indicative of conditions in the initial stages of PD activity. It is known that sustained discharge activity will alter the gaseous composition within the void and the gas pressure, which will have a significant impact on discharge mechanisms [16]. PD models have typically focused on the initial stages of PD activity and as such the assumptions they make can be assessed without considering changes to air composition.

For the purposes of this work a detailed model of plasma chemistry is not required and instead a drift diffusion model that considers electrons and 'abstract' positive and negative ions is implemented. This is an established approach and has been used in recent work on discharges in air in the literature [17, 18]. The intention is to develop a simple model that is still adequate for the purposes of the investigation. Qualitatively the plasma dynamics should agree with the established literature on discharges in bubbles in dielectric liquids that uses more complex models [12, 14]. As mentioned previously the difference between this work and the existing literature is the emphasis, and there is a discrepancy between the two areas of research in regards to the quantities of interest.

\section{Physical Concepts}

In this section the concepts that will be investigated in this work are outlined:

1. Plasma Dynamics - In earlier work the plasma dynamics of a PD in air was described as an electron avalanche transitioning into a positive streamer for a dielectric barrier discharge in a $3 \mathrm{~mm}$ air gap with an electric field slightly above the breakdown threshold of air [8. Simulations of discharges within spherical air bubbles in dielectric liquids, which are indicative of PD systems containing spherical voids, has shown that the plasma dynamics consisted of an electron avalanche moving against the applied electric field transitioning into a positive streamer moving with the electric field [12. Separate studies, at conditions that differ from a standard PD system, observed similar discharge dynamics [19, 20]. The earlier work of Niemeyer assumed that a PD consisted of a positive and negative streamer [5].

2. Inception and Residual Fields - Two widely used values in PD modelling are the inception and residual electric fields. For PD to occur the electric field in the void, typically the centre, must be at some inception value $E_{\text {inc }}$. For air filled voids $E_{\text {inc }}$ is typically set to

$$
E_{\text {inc }}=(E / p)_{\operatorname{cr}} p\left(1+\frac{B}{(p l)^{1 / 2}}\right)
$$


where $(E / p)_{\mathrm{cr}}=25.2 \mathrm{~V} \mathrm{~Pa}^{-1} \mathrm{~m}^{-1}$ is the constant of proportionality between the critical electric field $E_{\mathrm{cr}}$ required to sustain the discharge and the gas pressure $p, B=8.6 \mathrm{~Pa}^{1 / 2} \mathrm{~m}^{1 / 2}$ and $l$ is the length of the void in the direction of the discharge [3. It should be noted that in practice $(E / p)_{\text {cr }}$ will be dependent on streamer polarity. After the discharge has taken place, it is then assumed that the electric field in the void is reduced to a residual value which is equal to field in the streamer channel, $E_{\mathrm{ch}}$, and is proportional to $E_{\mathrm{cr}}$

$$
E_{\mathrm{res}}=E_{\mathrm{ch}}=\gamma E_{\mathrm{cr}}
$$

where $\gamma=0.35$, the average of 0.2 and 0.5 for positive and negative streamers respectively [3]. It should be noted that despite their widespread usage in the literature, since they were proposed by Niemeyer [3, 5], these equations may not necessarily be applicable to PD systems. In the case of the inception equation the experimental data for the ionisation parameters is determined from breakdown in air gaps between metallic electrodes [21, whereas in PD systems the discharge region is frequently bounded by a solid dielectric material. For the residual field equation, it is not clear that the field in the streamer channel will be equivalent to the field after the discharge has ceased. Furthermore, the conclusion that the channel field is proportional to the critical field appears to determined from metallic needle-plane experiments, 22, which have a highly divergent applied field, and do not have solid dielectric regions, which may limit the applicability of the result to certain PD systems. Another important point is that in the literature the term residual field has been used interchangeably with extinction field [6, 7]. This is slightly problematic as the term extinction voltage is often used to refer to the applied voltage at which repetitive discharge activity stops, [23, and its corresponding electric field does not have a clear relationship with $E_{\text {res }}$.

3. Surface Charge Distributions - It is commonly assumed that when the dielectric surfaces at each side of a discharge channel are symmetric, such as in spherical, cylindrical or ellipsoidal voids, the surface charge distribution at the dielectric surfaces are bipolar [5, 6, 24]. Earlier numerical studies and experimental work investigating PD in cylindrical voids suggest that the distribution is in fact not bipolar, [8, 25, and that the negative surface charge distribution has a greater spread due to the higher mobility of electrons compared to ions. Both of these studies have looked at PD for electric fields that just exceed the breakdown threshold. In previous models of PD activity it has been assumed that the PD surface charge is deposited directly over the surface charge of previous PDs [6. However, it is likely that field due to the charge of previous PDs will influence the charge deployed by subsequent PDs.

\section{Drift Diffusion Plasma Model}

The concepts listed in Section 2 will be critically tested by a dynamic plasma model. In this section the governing equations and boundary conditions are provided along with the model geometry under consideration. The model consists of three drift diffusion equations for three charged species, an electrostatic equation to determine the electric field and three Helmholtz equations to determine the photoionisation rate. The model is implemented in Comsol Multiphysics, [26, software which has been used to model discharges in air previously in the literature [17, 18].

\subsection{Geometry}

The model geometry is axisymmetric, consisting of a spherical void with a radius, $R_{\mathrm{v}}$, of $0.5 \mathrm{~mm}$ surrounded by epoxy resin between parallel plate electrodes. The motivation for this is that quasi-spherical voids often occur in epoxy resins in operating high voltage plant, due to errors in the curing process, which can facilitate PD activity. Furthermore the PD activity observed from this experimental arrangement is not significantly impacted by noise and as such a physical interpretation of discharge behaviour is possible [6]. The model geometry is shown in Figure 1. 


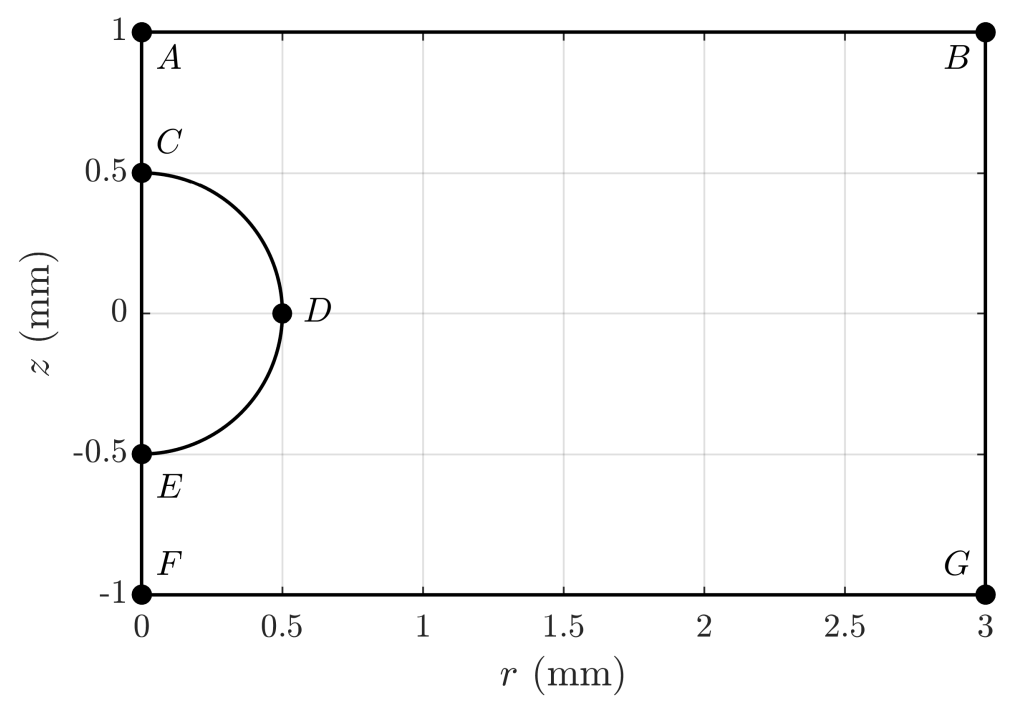

Figure 1: Model geometry with labelled vertices.

\subsection{Governing Equations}

The model solves drift diffusion equations for the number densities of electrons $n_{\mathrm{e}}$, positive ions $n_{\mathrm{p}}$ and negative ions $n_{\mathrm{n}}$

$$
\begin{aligned}
\frac{\partial n_{\mathrm{e}}}{\partial t}= & \alpha n_{\mathrm{e}}\left|\vec{W}_{\mathrm{e}}\right|-\eta n_{\mathrm{e}}\left|\vec{W}_{\mathrm{e}}\right|-\beta n_{\mathrm{e}} n_{\mathrm{p}} \\
& -\vec{\nabla} \cdot \vec{\Gamma}_{\mathrm{e}}+S_{\mathrm{ph}} \\
\frac{\partial n_{\mathrm{p}}=}{\partial t}= & \alpha n_{\mathrm{e}}\left|\vec{W}_{\mathrm{e}}\right|-\beta n_{\mathrm{n}} n_{\mathrm{p}}-\beta n_{\mathrm{e}} n_{\mathrm{p}} \\
& -\vec{\nabla} \cdot \vec{\Gamma}_{\mathrm{p}}+S_{\mathrm{ph}} \\
\frac{\partial n_{\mathrm{n}}}{\partial t}= & \eta n_{\mathrm{e}}\left|\vec{W}_{\mathrm{e}}\right|-\beta n_{\mathrm{n}} n_{\mathrm{p}}-\vec{\nabla} \cdot \vec{\Gamma}_{\mathrm{n}},
\end{aligned}
$$

where $S_{\mathrm{ph}}$ is the photoionisation rate and the corresponding fluxes, $\vec{\Gamma}_{\mathrm{e}}, \vec{\Gamma}_{\mathrm{p}}$ and $\vec{\Gamma}_{\mathrm{n}}$, are

$$
\begin{aligned}
\vec{\Gamma}_{\mathrm{e}} & =n_{\mathrm{e}} \vec{W}_{\mathrm{e}}-D_{\mathrm{e}} \vec{\nabla} n_{\mathrm{e}} \\
\vec{\Gamma}_{\mathrm{p}} & =n_{\mathrm{p}} \vec{W}_{\mathrm{p}} \\
\vec{\Gamma}_{\mathrm{n}} & =n_{\mathrm{n}} \vec{W}_{\mathrm{n}} .
\end{aligned}
$$

In equations 3 to $8 \vec{W}_{\mathrm{e}}, \vec{W}_{\mathrm{p}}$ and $\vec{W}_{\mathrm{n}}$ are the drift velocities for electrons, positive ions and negative ions respectively; $\alpha, \eta$ and $\beta$ are the ionisation, attachment and recombination coefficients respectively; $D_{\mathrm{e}}$ is diffusion coefficient for electrons. The swarm parameters introduced by Kang are used in this work [27, with the air pressure at atmospheric conditions. To find the electric field $\vec{E}$ the electrostatic equation is solved

$$
\nabla^{2} V=-\frac{\rho}{\varepsilon_{0} \varepsilon_{\mathrm{r}}}
$$

where $V$ is the electric potential,

$$
\vec{E}=-\vec{\nabla} V
$$

$\varepsilon_{0}$ is the absolute permittivity, $\varepsilon_{\mathrm{r}}$ is the relative permittivity of the material and $\rho$ is the volume charge density. The relative permittivity of air is set to 1 , and the relative permittivity of epoxy is set to 4.4. $\rho$ is 
related to the charge carriers as follows

$$
\rho=e\left(n_{\mathrm{p}}-n_{\mathrm{e}}-n_{\mathrm{n}}\right),
$$

where $e$ is the charge of an electron. The use of three charged species, electrons and 'abstract' positive and negative ions, to simulate discharges in air has been widely used in the literature [17, 18, 28, 29.

To calculate the photoionisation rate the three-exponential Helmholtz model developed by Bourdon et al was used [28. This approximates the computationally expensive model proposed by Zheleznyak et al [30]. The photoionisation rate, $S_{\mathrm{ph}}$, is approximated as

$$
S_{\mathrm{ph}}=\sum_{i=1}^{3} S_{\mathrm{ph} i}
$$

where each $S_{\mathrm{ph} i}$ solves a Helmholtz equation

$$
\nabla^{2} S_{\mathrm{ph} i}-\left(\lambda_{i} p_{\mathrm{O}_{2}}\right)^{2} S_{\mathrm{ph} i}=-A_{i} p_{\mathrm{O}_{2}}^{2} S
$$

with constants $\lambda_{i}$ and $A_{i}$ given in Table 1, $p_{\mathrm{O}_{2}}$ is the partial pressure of oxygen (150 Torr at atmospheric pressure) and

$$
S=\xi I
$$

where

$$
I=\alpha n_{\mathrm{e}}\left|\vec{W}_{\mathrm{e}}\right|
$$

and $\xi=0.00228$ for the physical conditions under consideration [28, 31. $S$ will be referred to as the photoionisation source and is directly proportional to the collisional ionisation rate, $I$. [28].

\begin{tabular}{ccc}
$i$ & $A_{i}\left(\mathbf{m}^{-\mathbf{2}} \mathbf{T o r r}^{-\mathbf{2}}\right)$ & $\lambda_{i}\left(\mathbf{m}^{\mathbf{- 1}} \mathbf{T o r r}^{\mathbf{- 1}}\right)$ \\
\hline \hline 1 & 1.98 & 5.53 \\
2 & 51 & 14.6 \\
3 & 4886 & 89
\end{tabular}

Table 1: Helmholtz Equation Parameters from [28]

\subsection{Boundary Conditions}

Boundary condition for the drift-diffusion equations are required at the boundaries of the void, they are set as follows

$$
\begin{aligned}
& \vec{n} \cdot \vec{\Gamma}_{\mathrm{e}}=\vec{n} \cdot\left(a_{\mathrm{e}} n_{\mathrm{e}} \vec{W}_{\mathrm{e}}-\gamma n_{\mathrm{p}} \vec{W}_{\mathrm{p}}\right) \\
& \vec{n} \cdot \vec{\Gamma}_{\mathrm{p}}=\vec{n} \cdot a_{\mathrm{p}} n_{\mathrm{p}} \vec{W}_{\mathrm{p}} \\
& \vec{n} \cdot \vec{\Gamma}_{\mathrm{n}}=\vec{n} \cdot a_{\mathrm{n}} n_{\mathrm{n}} \vec{W}_{\mathrm{n}}
\end{aligned}
$$

where $\gamma$ is the ion-impact secondary emission coefficient,

$$
a_{i}= \begin{cases}1 & \text { if } \operatorname{sgn}\left(q_{i}\right)=\operatorname{sgn}(\vec{n} \cdot \vec{E}) \\ 0 & \text { otherwise }\end{cases}
$$

$i=\mathrm{e}, \mathrm{p}$ and $\mathrm{n}$, sgn is the sign function, $q_{i}$ is the charge of the $i$ th species and $\vec{n}$ is the outwards facing normal unit vector at the void surface, these boundary conditions have been used to describe dielectric boundaries in earlier work [18. The secondary ionisation coefficient at the void surface was set to a token value of 0.001, it should be noted that the secondary ionisation coefficient has yet to be accurately determined for a large number of dielectric materials. 16 neglects incoming thermal flux of charged particles which has been considered in other work [32. However, as the drift velocity is significantly higher than the thermal velocity 
for the conditions under consideration, this is not thought to impact the findings of this work, assuming that electron temperatures are at most within an order of magnitude of $1 \mathrm{eV}$.

Boundary conditions are also required for $V$. These are largely trivial with the exception of the void surface, where charge will be deployed due to the discharge. The surface charge density $\sigma$ at the void surface can be calculated through

$$
\sigma=\int_{0}^{t} e \vec{n} \cdot\left(n_{\mathrm{p}} \vec{W}_{\mathrm{p}}-n_{\mathrm{e}} \vec{W}_{\mathrm{e}}-n_{\mathrm{n}} \vec{W}_{\mathrm{n}}\right) d t,
$$

an approach used widely used in the literature [18, 32. The boundary condition for $V$ at the void boundary is

$$
\vec{n} \cdot\left(\vec{D}_{1}-\vec{D}_{2}\right)=\sigma
$$

where $\vec{D}$ is the electric displacement field,

$$
\vec{D}=\varepsilon_{0} \varepsilon_{r} \vec{E},
$$

and the 1, 2 subscripts denote evaluation in regions either side of a surface with normal vector $\vec{n}$ pointing from region 1 to region 2. In the system under consideration a high voltage electrode, at potential $V_{0}$, is located at the top boundary of the geometry and a ground electrode is located at the bottom. Combining this with conditions for the axisymmetric and far field boundaries completes the boundary conditions for $V$.

In the original integral model of photoionisation perfect absorption at boundaries is assumed [30]. In the work of Bourdon et al the external boundaries of the system were effectively in the far field region [28]. At these boundaries $S_{\mathrm{ph} 1}$ was set equal to the the photoionisation rate calculated using the integral model, with $S_{\mathrm{ph} 2}=S_{\mathrm{ph} 3}=0$. This is because $S_{\mathrm{ph} 1}$ corresponds to the smallest value of $\lambda_{i}$, Table 1 which means that it is the component with the longest photoionisation range so is likely to be the dominant component in the far-field region. For the arrangement considered here the void boundaries cannot be considered as far field; photoionisation sources will be very close to the void surface. This means that the assumption that $S_{\text {ph } 1}$ dominates at the boundary is not valid, furthermore the fine numerical mesh required at the boundaries means that calculating $S_{\mathrm{ph}}$ from the integral model at the void boundary is computationally expensive. In this system, the dominant Helmholtz component at the boundaries is likely to be the component with the largest source term, corresponding to the largest $A_{j}$, which is $S_{\mathrm{ph} 3}$. It was found that employing Dirichlet boundary conditions on $S_{\mathrm{ph} 1}$ and $S_{\mathrm{ph} 2}$

$$
S_{\mathrm{ph} 1}=S_{\mathrm{ph} 2}=0 \text { at the void boundary }
$$

and Neumann boundary conditions on $S_{\mathrm{ph} \mathrm{3}}$,

$$
\vec{n} \cdot \vec{\nabla} S_{\text {ph } 3}=0 \text { at the void boundary }
$$

where $\vec{n}$ is a normal vector at the boundary, gave results that were in good agreement with the full integral model of photoionisation. It should be noted that these boundary conditions are based on empirically fitting the Helmholtz model to the full integral model, and are not indicative of the physical process of photoionisation. This was determined by comparing the full integral model against the Helmholtz model. The collisional ionisation rate $I$ is set to a Gaussian distribution centred on the $z$ axis

$$
I=I_{0} \exp \left(-\left(r^{2}+\left(z-z_{0}^{2}\right)\right) / \delta^{2}\right),
$$

where $I_{0}=10^{31} \mathrm{~m}^{-3} \mathrm{~s}^{-1}, z_{0}$ is the $z$ coordinate of the centre of distribution and $\delta_{0}$ is a spatial parameter governing the spread of the distribution. Two cases are considered, $z_{0}=0 \mathrm{~mm}$, ionisation taking place in the centre of the void, and $z_{0}=0.5 \mathrm{~mm}$, ionisation taking place at the void boundary. $\delta=0.1 \mathrm{~mm}$ in both cases, this is analogous to the approach used in [28. A comparison of the photoionisation rate along the $z$ axis of the void using the two different models is shown in Figure2. It can be seen that the agreement is fairly good from an order of magnitude perspective with some notable discrepancies. 


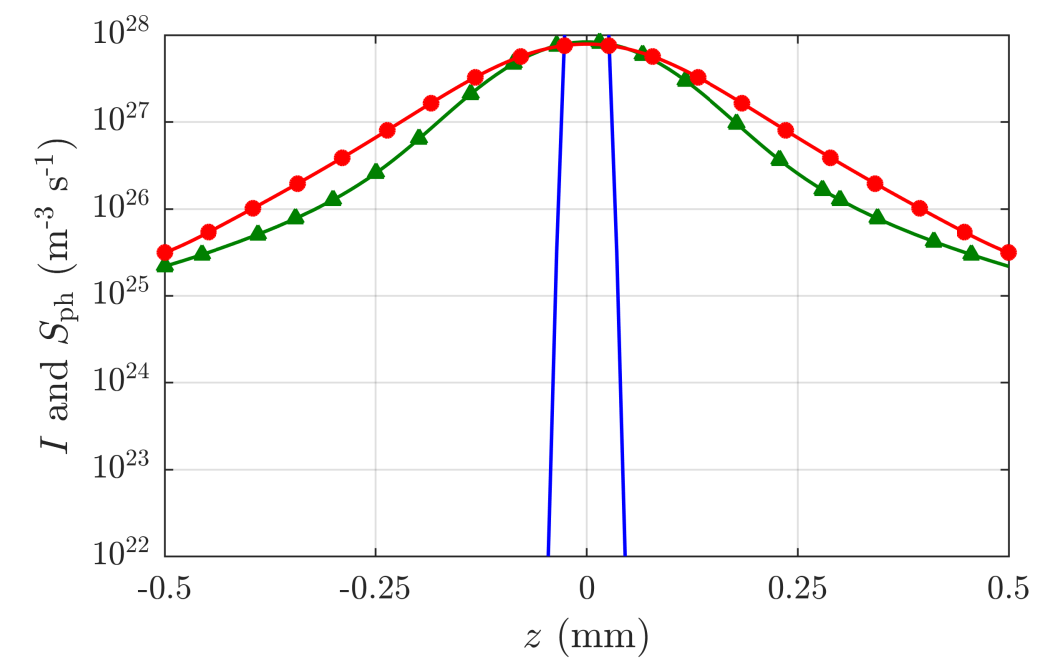

$I$ - Collisional Ionisation Rate $\smile-S_{\mathrm{ph}}-$ Full Model $\multimap-S_{\mathrm{ph}}-$ Helmholtz

(a)

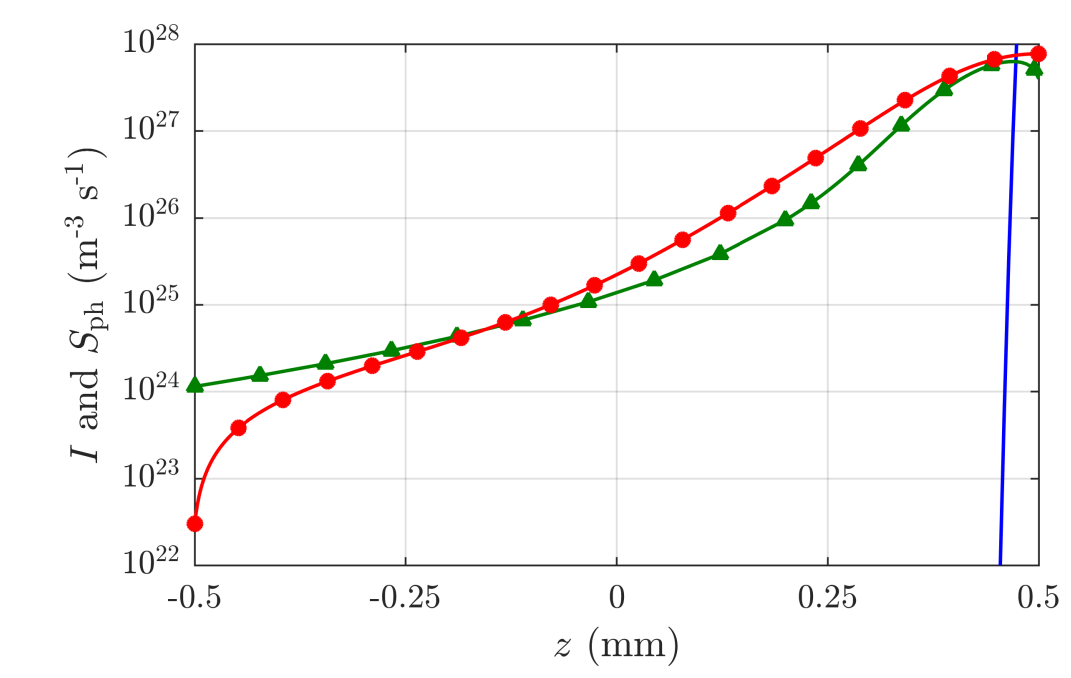

$\longrightarrow I$ - Collisional Ionisation Rate $\multimap S_{\mathrm{ph}}-$ Full Model $\multimap S_{\mathrm{ph}}-$ Helmholtz

(b)

Figure 2: Comparison of photoionisation rate calculated using full model of photoionisaton and Helmholtz model with modified boundary conditions. The Gaussian collisional ionisation production rate was centred at: (a) $z_{0}=0 \mathrm{~mm}$ and (b) $z_{0}=0.5 \mathrm{~mm}$

The absolute error between the full and Helmholtz models is shown in Figure 3. The error is highest at the void boundary, $z_{0}=0.5 \mathrm{~mm}$, with an error of $3.7 \times 10^{27} \mathrm{~m}^{-3} \mathrm{~s}^{-1}$. It should be noted however that in this region the collisional ionisation, $I$, is dominant, with a value of $10^{31} \mathrm{~m}^{-3} \mathrm{~s}^{-1}$ at the void boundary and photoionisation is a relatively insignificant process. It is not thought that these discrepancies will significantly impact the findings presented in this work as they are all roughly within an order of magnitude, which was the case with the original work where this technique was successfully applied [28]. Furthermore, numerical investigations by the authors also suggest that order of magnitude changes to photoionisation rates will not 
impact the current and charge distributions with are the quantities of interest in this work.

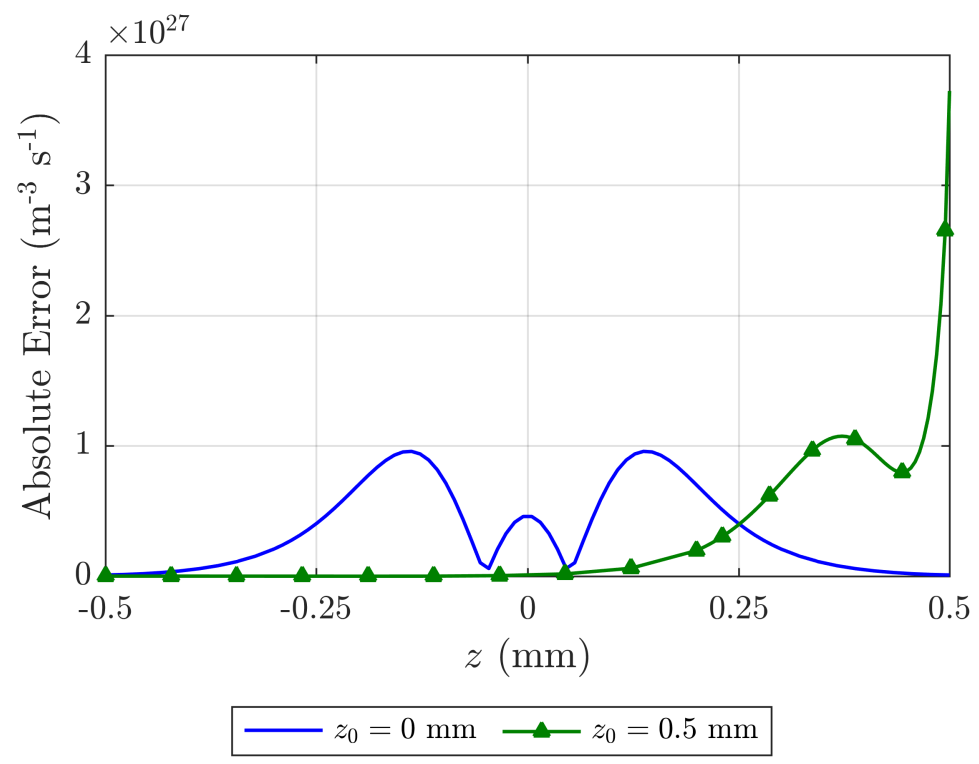

Figure 3: Absolute error between the photoionisation rate calculated using the full model and Helmholtz model. In the region close to the void boundary where the error is largest the collisional ionisation rate dominates photoionisation.

A summary of the boundary conditions used are shown in Table 2. Boundaries are defined using the vertices in Figure 1. It should be noted that the drift diffusion and Helmholtz equations are only solved inside the void region and as such they only require boundary conditions at the void surface.

\begin{tabular}{|c|c|c|c|c|c|c|c|}
\hline Boundary & $V$ & $n_{\mathrm{e}}$ & $n_{\mathrm{p}}$ & $n_{\mathrm{n}}$ & $S_{\mathrm{ph} 1}$ & $S_{\mathrm{ph} 2}$ & $S_{\mathrm{ph} 3}$ \\
\hline$A B$ & $V_{0}$ & - & - & - & - & - & - \\
\hline$C E$ & $\frac{\partial V}{\partial r}=0$ & $\frac{\partial n_{\mathrm{e}}}{\partial r}=0$ & $\frac{\partial n_{\mathrm{p}}}{\partial r}=0$ & $\frac{\partial n_{\mathrm{n}}}{\partial r}=0$ & $\frac{\partial S_{\mathrm{ph} 1}}{\partial r}=0$ & $\frac{\partial S_{\mathrm{ph} 2}}{\partial r}=0$ & $\frac{\partial S_{\mathrm{ph} 3}}{\partial r}=0$ \\
\hline$C D, D E$ & $\vec{n} \cdot\left(\vec{D}_{1}-\vec{D}_{2}\right)=\sigma$ & 16 & 17 & 18 & $S_{\mathrm{ph} 1}=0$ & $S_{\mathrm{ph} 2}=0$ & $\vec{n} \cdot \vec{\nabla} S_{\mathrm{ph} 3}=0$ \\
\hline$A C, E F$ & $\frac{\partial V}{\partial r}=0$ & - & - & - & - & - & - \\
\hline$B G$ & $\vec{n} \cdot \vec{D}=0$ & - & - & - & - & - & - \\
\hline$F G$ & 0 & - & - & - & - & - & - \\
\hline
\end{tabular}

Table 2: Boundary Conditions

\subsection{Initial Conditions}

Due to their explicit time dependence initial conditions are required for the drift diffusion equations. The initial conditions for the electron and positive ion number densities were set to be a Gaussian seed charge, with a magnitude insufficient to distort the applied field. This approach has been widely used when modelling breakdown in air using drift diffusion equations [12, 18, 28. The initial number of negative ions was set to zero. Explicitly the initial conditions for the number densities are

$$
\begin{aligned}
\left.n_{\mathrm{e}, \mathrm{p}}\right|_{t=0} & =n_{0} \exp \left(-\left(r^{2}+\left(z-z_{0}^{2}\right)\right) / s_{0}^{2}\right) \\
\left.n_{\mathrm{n}}\right|_{t=0} & =0
\end{aligned}
$$


where $n_{0}=10^{11} \mathrm{~m}^{-3}, z_{0}=0.5 \mathrm{~mm}$ and $s_{0}=25 \mu \mathrm{m}$. Order of magnitude variations in the parameters defining the initial distribution, $n_{0}, z_{0}$ and $s_{0}$, were only found to impact the time taken for discharges to begin, and did not impact the findings of the work presented in Section 4 .

\subsection{Measurable Quantities}

For PD activity under AC conditions a PD is typically quantified by its angle of occurrence in the $\mathrm{AC}$ cycle, known as phase angle, and the change in charge on a suitable measuring electrode, known as the apparent charge [6. In this work single discharges are investigated at a range of applied voltages, so the phase angle is not a quantity of interest. The purpose of this work is to assess the simplifying assumptions used to describe a single discharge. For the system under consideration the measuring electrode is assumed to the the ground electrode, the apparent charge, $q^{\prime}$, of the discharge is

$$
q^{\prime}=\int_{S} \vec{n} \cdot\left(\left.\vec{D}\right|_{t=t_{\mathrm{f}}}-\left.\vec{D}\right|_{t=t_{0}}\right) d A
$$

where $t_{0}$ is the time immediately before the discharge takes place, $t_{\mathrm{f}}$ is the time the discharge finishes, $S$ is the ground electrode surface and $\vec{n}$ is an outwards facing normal unit vector. Comparing the PD system under investigation with PD measurements in similar systems it is expected that PD magnitudes will be in the order of $100 \mathrm{pC}\left[3,6\right.$. The apparent current pulse $I^{\prime}$ on the measuring electrode can also be calculated

$$
I^{\prime}=\int_{S} J^{\prime} d A
$$

where $J^{\prime}$ is the apparent current density on the measuring electrode surface defined as

$$
J^{\prime}=\frac{\partial}{\partial t}(\vec{n} \cdot \vec{D}) .
$$

Measurements of the current pulse $I^{\prime}$, for PD systems similar to the that under investigation, have a timescale in the order of nanoseconds with a sharp rise followed by a relatively long decay [16].

\subsection{Model Configurations}

In order to test the concepts, outlined in Section 2, it is necessary to run the model in two different configurations as follows:

(A) Discharges in Virgin Voids - It is proposed to use the model to examine the physics of PD in virgin voids, i.e. $\sigma_{t=0}=0$, at a range of applied voltages. By gradually increasing the applied voltage until a discharge occurs, the inception field for the void can be determined and compared against the value calculated using (1). By considering the field after the discharge the residual field can be determined, which can then be compared to the value calculated using (2). The plasma dynamics and the surface charge distributions after PD can also be investigated. Due to the similarity of the system it is expected that plasma dynamics will be similar to those observed in simulation of discharges in bubbles in liquid dielectrics. A qualitative comparison with this work, [12, will establish whether the model is accurately describing the discharge process. The model is set to have a constant applied voltage for the duration of the discharge even though in reality the discharges are occurring in an AC system with a varying applied voltage. This is because the discharges take place over nanoseconds, whereas the applied voltage frequencies of interest are typically $50-60 \mathrm{~Hz}$. The purpose of this work is to assess the simplifying assumptions used to represent a single discharge in PD activity models. The variation in the applied voltage over the time scale of the discharge is negligible.

(B) Effect of Subsequent Discharges - The resulting surface charge distribution from a discharge in a virgin void will be used as an initial condition for a second discharge. This will provide insight into the physics of PD in an electric field that is distorted by the surface charge, and also see how the charge deployed by the second PD compares to the first. 


\subsection{Implementation}

The model is implemented in Comsol Multiphysics 5.3 using the in-built physics libraries [26]. Six physics libraries were used; three transport of diluted species libraries to solve (3)-(5); an electrostatics library to solve (9); a coefficient form PDE library to solve (13) and a boundary ODE libary to solve (20). In the transport of diluted species libraries the consistent stabilisation techniques of streamline and crosswind diffusion were used [33 35]. This approach was used in a previous the model, from which this model was developed, and is similar to recent work in literature [17, 18].

Comsol uses method of lines to convert the PDEs into a set ODEs. The ODEs are solved by an implicit solver that automatically chooses the interpolation order in time. The variable-order variable step-size backward differentiation formulae (BDF) are used for implicit time-stepping with the non-linear controller activated, in conjunction with the PARDISO direct solver. The libraries were solved for independently in a segregated arrangement as it was found to vastly reduce the computational time and improved the numerical stability of the solution. Spatial discretization was linear order for the charged species number densities, and quadratic order for all other dependent variables. A maximum time step of $0.01 \mathrm{~ns}$ was enforced throughout the simulation. The maximum calculated Courant numbers based on the streamer velocity were below 4 . These values appear at the times when the streamer enters the region with fine mesh close to the boundary. The implicit scheme used by COMSOL is able to dealt with such high Courant numbers by introducing artificial numerical diffusion. The overall effect of that is a slight broadening of sharp gradients, which does not affect the conclusions of the paper on the surface charge density distribution and the electric in the void.

The final step in implementing the model is the creation of a numerical mesh. From numerical experiments with a one dimensional model it was found that sharp gradients in the charged species number densities were present at the void boundary, in particular in regions close to the applied voltage boundary and provided a guide for meshing the two dimensional model geometry. The mesh was created using Comsol's automatic meshing algorithms, with the element size controlled by limiting the maximum size. It was found that smooth streamer propagation could be achieved with a maximum element size of $1 \mu \mathrm{m}$ within $0.1 \mathrm{~mm}$ of the void boundary for regions near the top of the void. For the remaining regions within $0.1 \mathrm{~mm}$ of the void the maximum element size was set to $2 \mu \mathrm{m}$, and the maximum element size of the rest of the void set to $5 \mu \mathrm{m}$. In order to accurately calculate the apparent charge a reasonably fine mesh is also required in the epoxy resin region, this was achieved by restricting the maximum element size to $30 \mu \mathrm{m}$. The full mesh consisted of 272960 triangular elements and is shown in Figure 4 . Simulations using a mesh refined by a factor of 2 in all regions resulted in a change within $5 \%$ to the quantities of interest. 


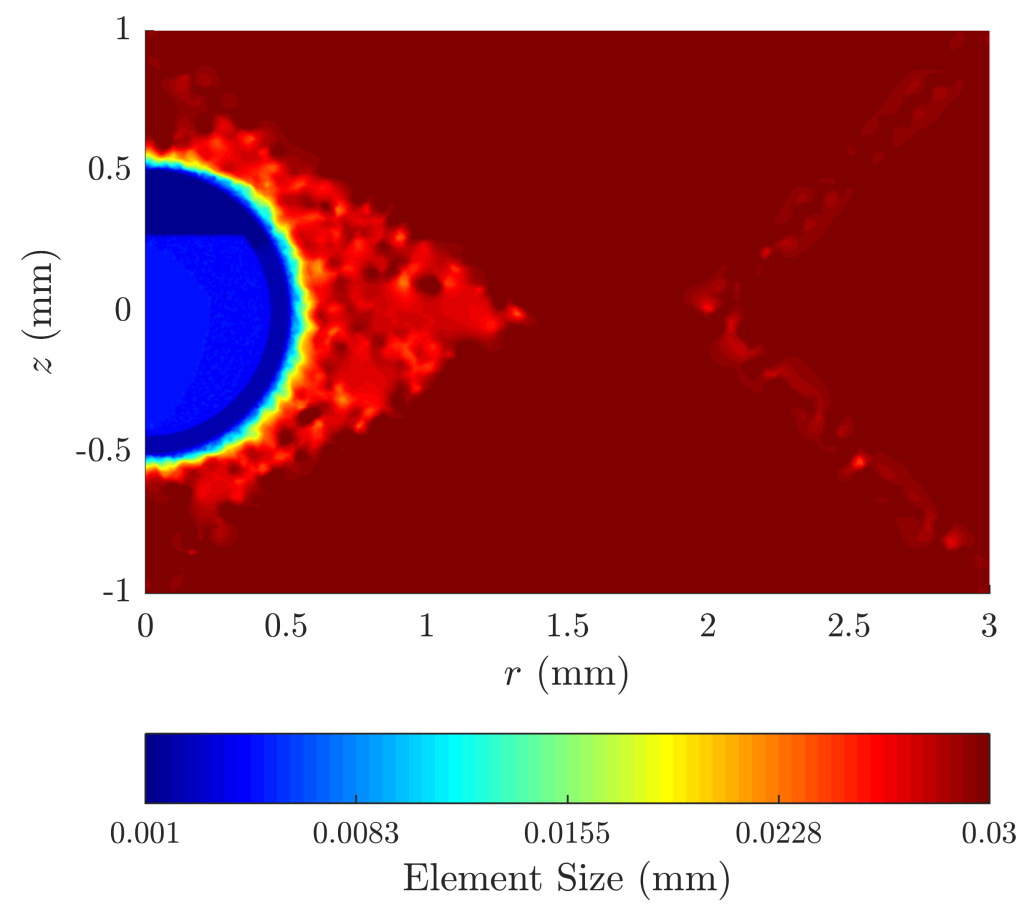

Figure 4: Element size surface plot of the numerical mesh used for the simulations. Mesh scaling was developed based on the plasma dynamics observed.

The model was solved on $162.6 \mathrm{GHz}$ Intel Sandybridge processors and had a run time in the order of a day.

\section{Results and Discussion}

Three different model configurations are considered in this work; Discharges in Virgin Voids; Effect of Subsequent Discharges and Post-Discharge Plasma Dynamics. The results for each configuration are presented in this section, their implications discussed. It is important to realise that in this work PD is investigated in atmospheric pressure air. After sustained PD activity it is well known that changes to gas composition, pressure and void surface chemistry can take place, and that this will significantly influence plasma dynamics [16, 36. As such, these results are applicable to initial stage of discharge activity, which could last in the order of a thousand $50 \mathrm{~Hz} \mathrm{AC}$ cycles.

\subsection{Discharges in Virgin Voids}

The model was used to investigate discharges in virgin voids. A range of applied voltages, $V_{0}$ in Table 2 with negative polarity in increments of $-0.5 \mathrm{kV}$, were considered. The applied voltages was set to a negative polarity so that the apparent charge and current was positive. Due to the symmetry of the geometry and governing equations an applied voltage with the same magnitude and positive polarity would simply have resulted in identical results to those at negative polarity undergoing a reflection in the central plane, $z=0$. In other words the dynamics of the discharge will simply be reversed due to a change in voltage polarity. The anti-symmetry of PD activity under changes in voltage polarity in systems of this type, discharges in gaseous voids surrounded by dielectric material, under AC conditions is observed experimentally [3, 6].

The dynamics of the discharges were the same at all applied voltages, therefore for the sake of brevity figures showing the dynamics of the charged species will only be provided for the discharge at $9 \mathrm{kV}$. The 
results are similar to dynamics of dielectric barrier discharges described elsewhere in the literature, [8, 19], and are very similar to the discharges investigated in [12. The dynamics of the discharge can be split into approximately four stages (I-IV) :

(I) - 0 to 5 ns - The discharge begins with the Gaussian seed charge moving to the bottom of the void, see Figure 5. The local field is initially insufficient to distort the applied field, see Figure 5 .

(II) - 5 to $10 \mathrm{~ns}$ - At the bottom of the void the charge multiplies, with the number densities growing to peak values of $10^{19} \mathrm{~m}^{-3}$, see Figure 6 at this time negative charge deployed into the bottom of the void leads to a change in surface charge density, see Figure 9. The local field due to the charged species is now noticeable, see Figure 6r. Significant changes to the location and magnitude of the initial seed charge was only found to influence the length of time of the multiplication phase, no other noticeable impact on the plasma dynamics was observed.

(III) - 10 to $20 \mathrm{~ns}$ - The number densities then grow to such an extent that a positive streamer is formed, moving towards the top of the void. The number densities of charged species reach peak values of $10^{21} \mathrm{~m}^{-3}$, and the local fields due to the space charge is significantly higher than the applied field, see Figure 7. The positive streamer reaching the top of the void, between 15 and $20 \mathrm{~ns}$, leads to an increase in the positive surface charge density on the top surface of the void, see Figure 9. The positive charge spot has a higher peak surface charge density, but is less diffused than the negative charge spot, see Figure 9. The surface charge density distributions for $9.5 \mathrm{kV}$ and $10 \mathrm{kV}$ are essentially scaled distributions of those at $9 \mathrm{kV}$. Similar distributions were also observed in earlier simulation work, [8], and in Pockel's cell measurements of surface charge density from discharges in air gaps bounded by a dielectric material below and a metallic electrode above [25].

(IV) - After 20 ns - After the positive streamer has reached the top of the void the local electric field from the volume charge density begins to decrease and the surface charge density deployed by the discharge is sufficient to nearly completely "short" the electric field inside the void except in regions close to the top surface of the void, see Figures 8; and 11. At the top surface of the void, deployment of positive surface charge continues at a slow rate, due to the low values of positive ion flux, due to low values of the electric field and consequently positive ion velocity. After hundreds of nanoseconds the deployment of positive surface charge stops, and the electric field is "shorted" in all regions in the void. The discharge now enters the charge recombination phase, which occurs over much longer timescales. 


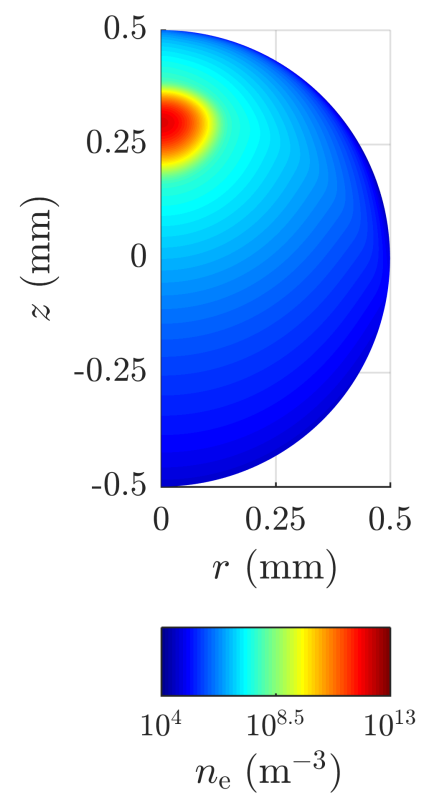

(a)
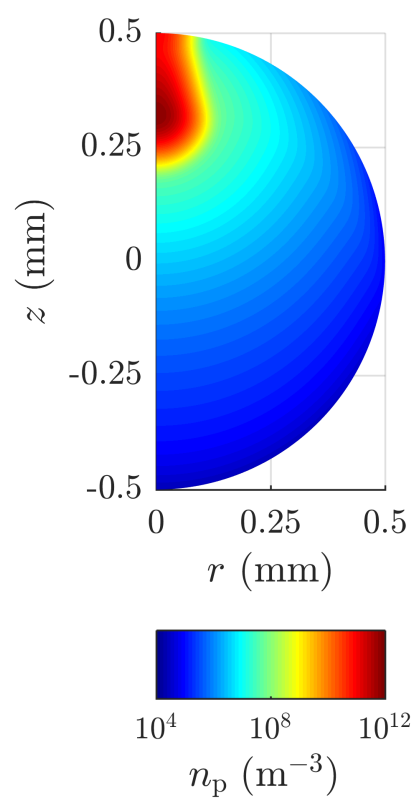

(b)
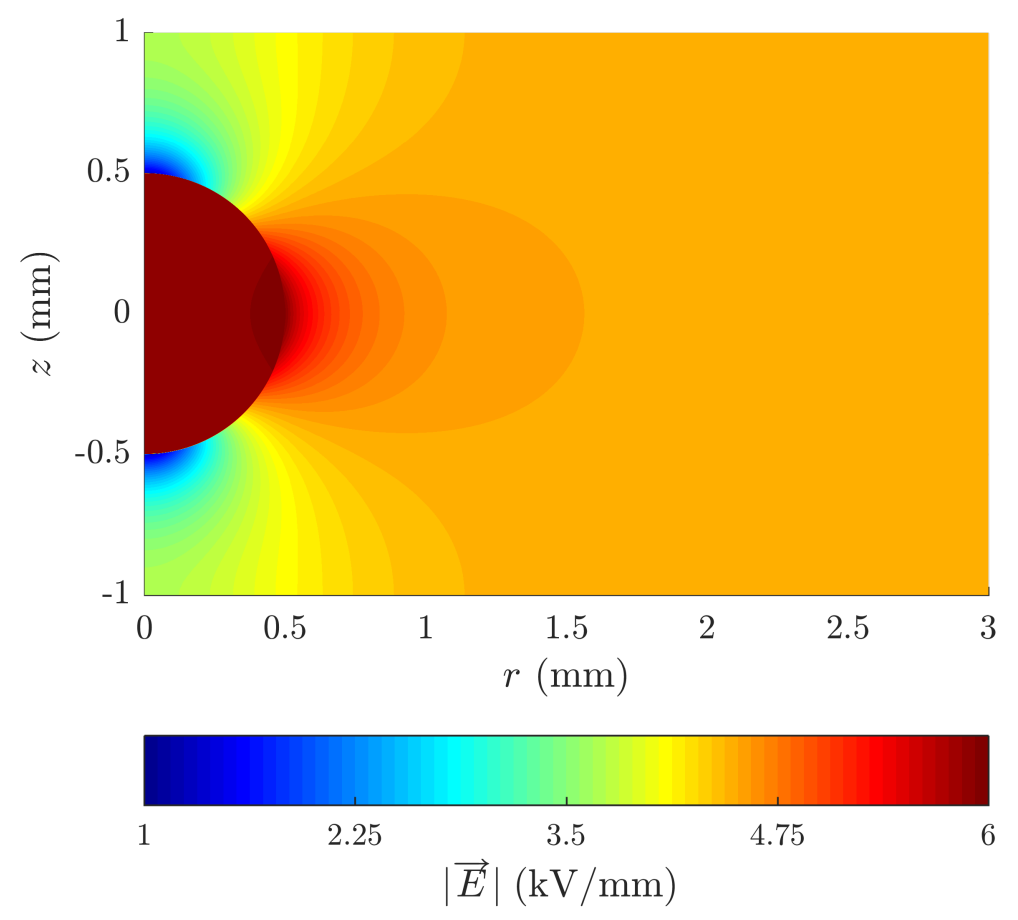

(c)

Figure 5: Discharge dependent variables at $1 \mathrm{~ns}$ (I) (a) electron number density, (b) positive ion number density, (c) electric field magnitude. 


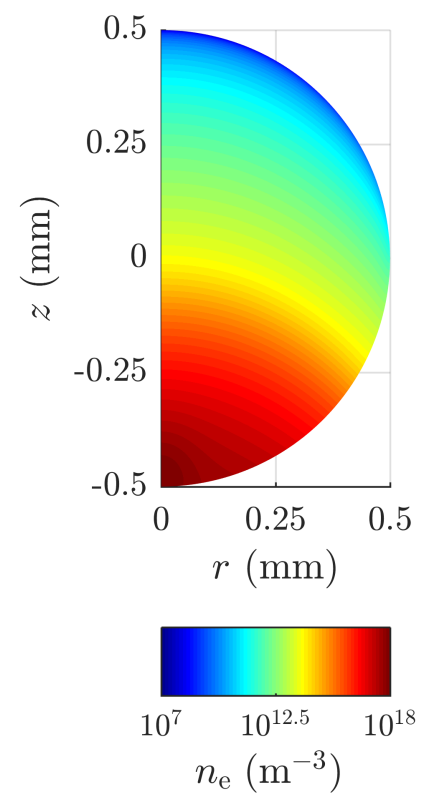

(a)
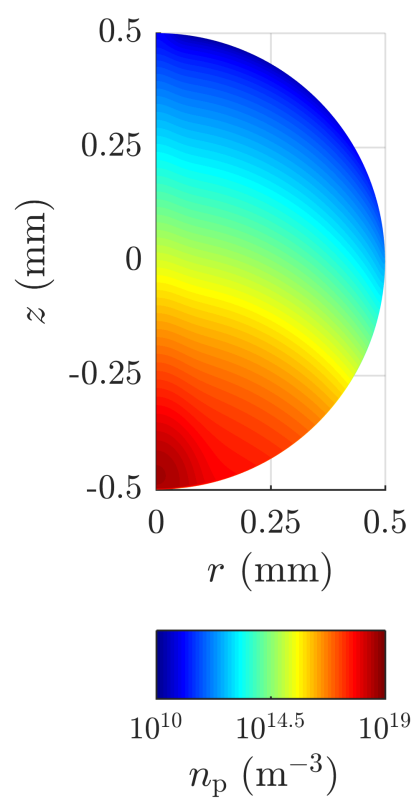

(b)
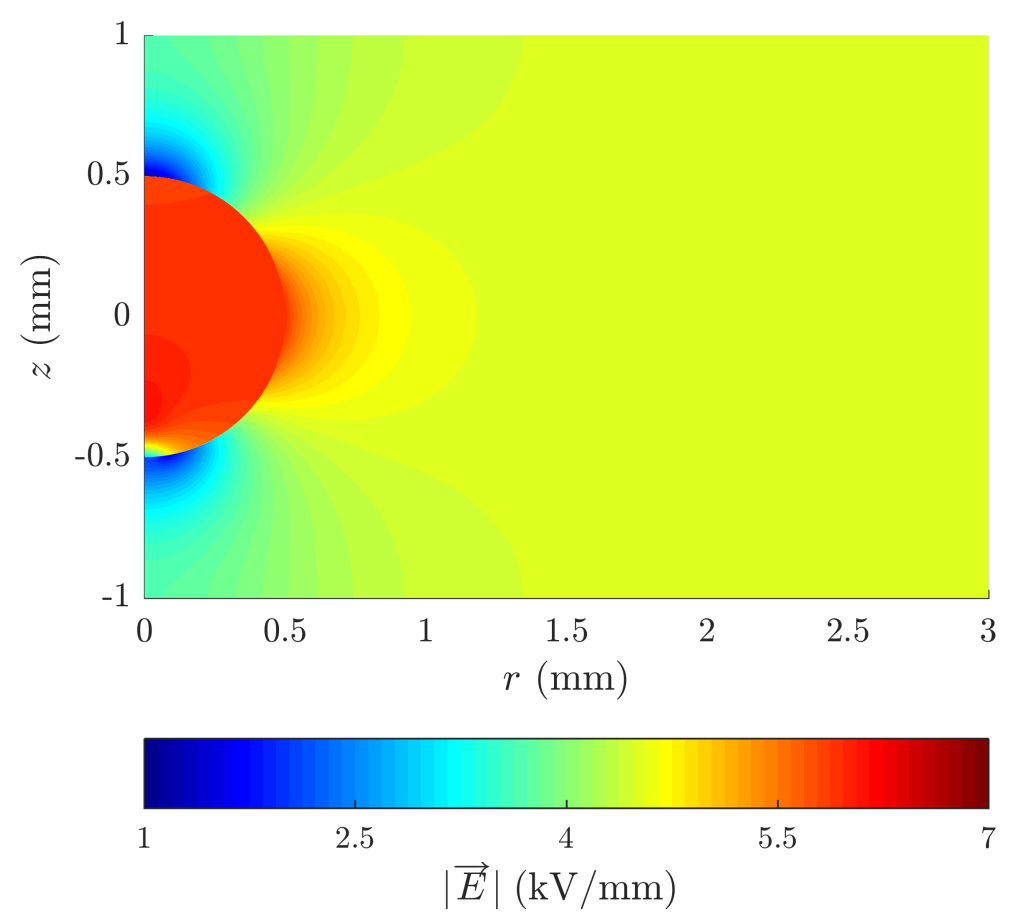

(c)

Figure 6: Discharge dependent variables at $7 \mathrm{~ns}$ (II) (a) electron number density, (b) positive ion number density, (c) electric field magnitude. 

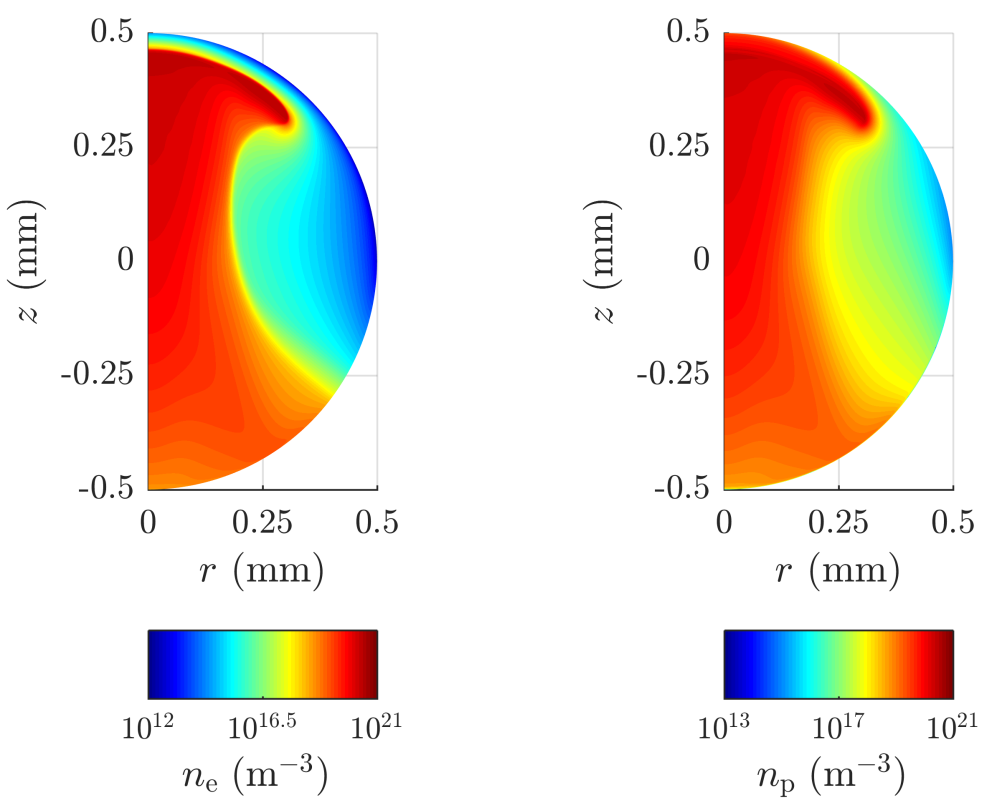

(a)

(b)

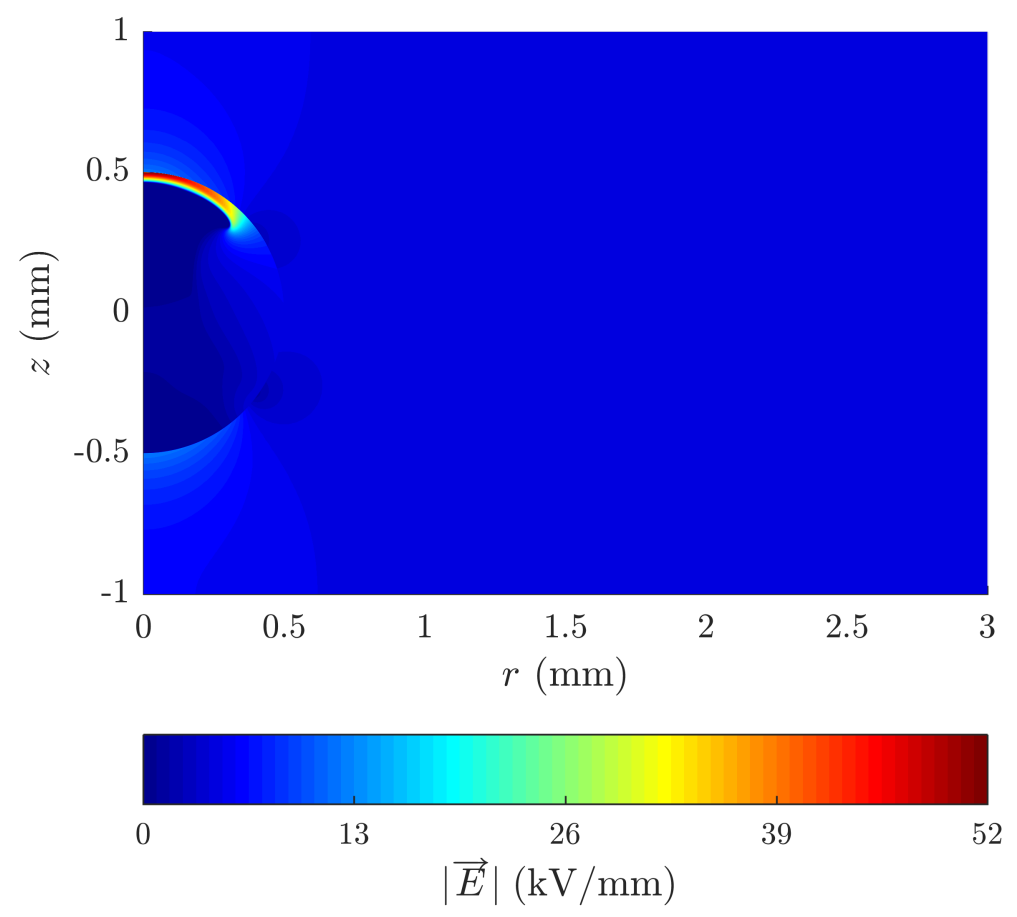

(c)

Figure 7: Discharge dependent variables at $15 \mathrm{~ns}$ (III) (a) electron number density, (b) positive ion number density, (c) electric field magnitude. 

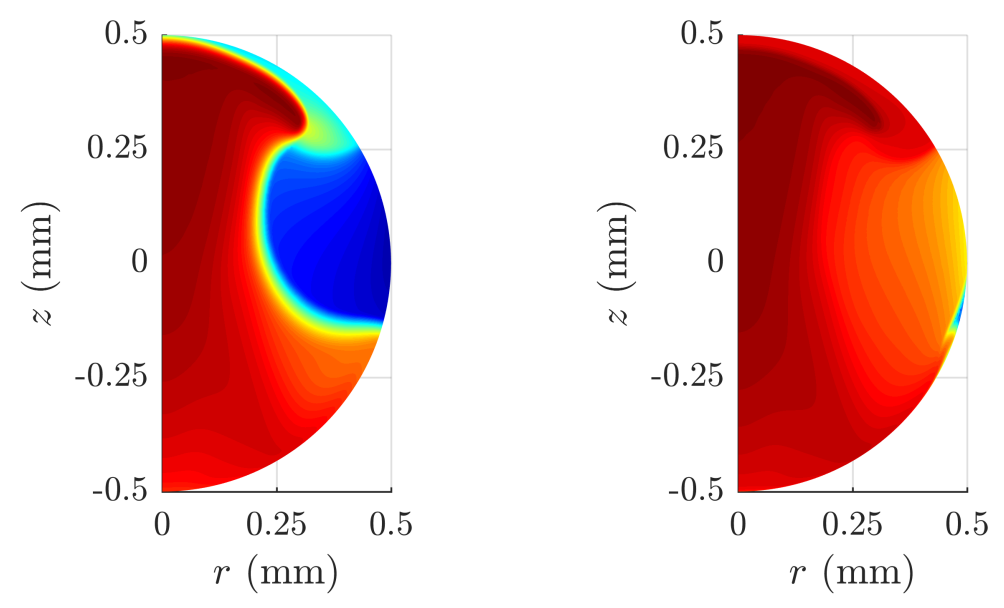

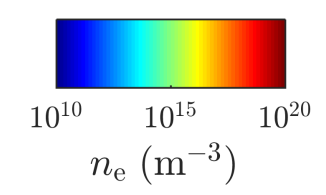

(a)

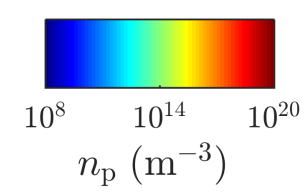

(b)

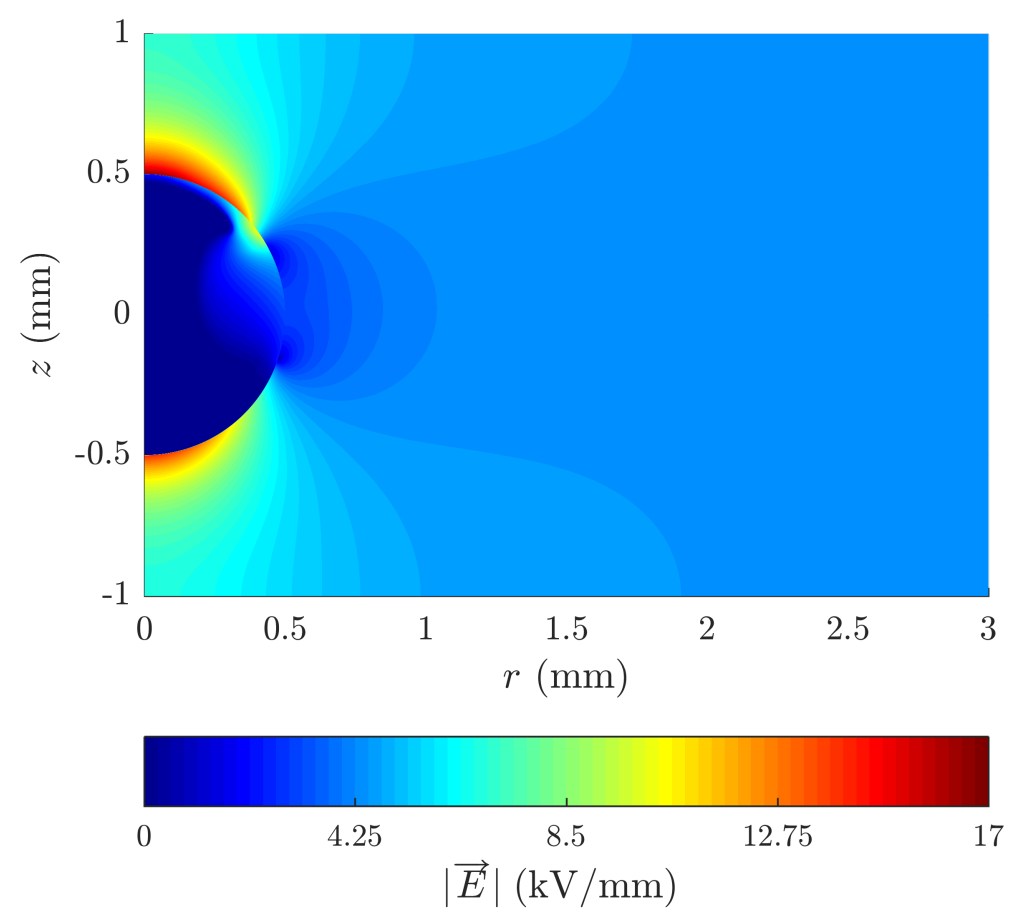

(c)

Figure 8: Discharge dependent variables at $60 \mathrm{~ns}$ (IV); (a) electron number density, (b) positive ion number density, (c) electric field magnitude. 


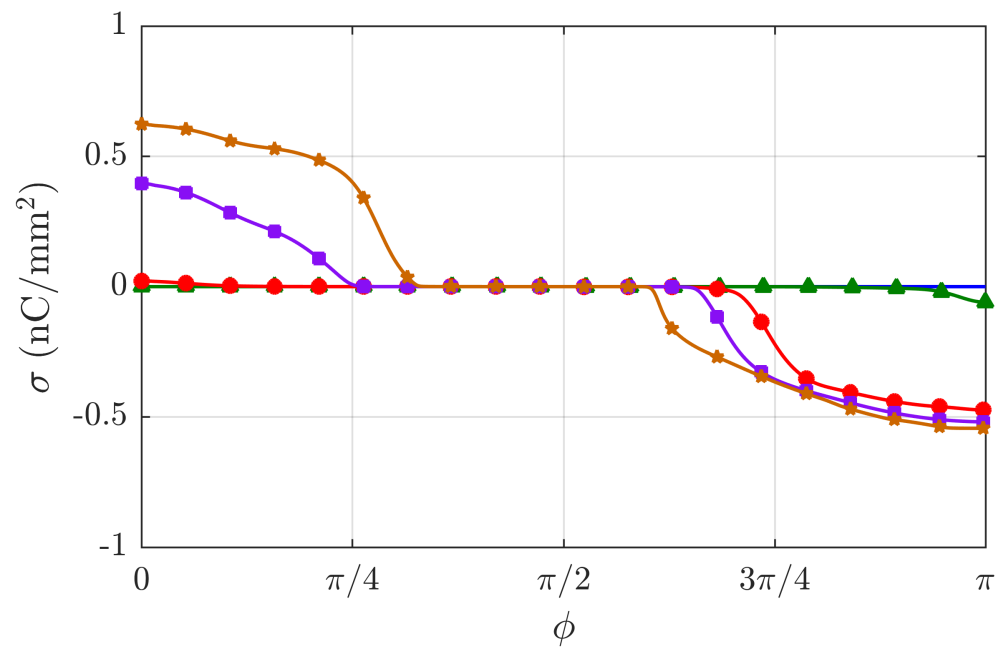

$\longrightarrow 1 \mathrm{~ns}-\mathrm{I} \longrightarrow 6 \mathrm{~ns}-\mathrm{III} \longrightarrow \star 60 \mathrm{~ns}-\mathrm{IV}$
$\longrightarrow 7 \mathrm{~ns}-\mathrm{II} \longrightarrow 20 \mathrm{~ns}-\mathrm{IV}$

Figure 9: Polar plot of surface charge density on the void surface, with polar angle $\phi$, at different times during the discharge at $9 \mathrm{kV}$. The stages corresponding to each time are also provided.

It also of interest to compare the standard measurable quantities of the discharges, apparent charge and current, at different applied voltages. The apparent charges are shown in Table 3. They display an approximately linear increase with the applied voltage. This is as expected, because the discharge deploys surface charge at the boundaries of the void to oppose the applied field. As the applied voltage increases the local electric field from the discharge will increase to oppose it and there is a linear relationship between charge and its corresponding electric field. The magnitude of the discharges were in the range of $100 \mathrm{pC}$, which is in good agreement with existing measurements in the literature [3, 6. An earlier published version of the model, [37, with a geometry based on an experimental setup in the literature, so a direct comparison with experimental data was possible, gave good agreement with the measured apparent charge magnitudes, with a simulated apparent charge magnitude of $546 \mathrm{pC}$ for experimental apparent charge magnitudes in the range of 500-600 pC, which is within the uncertainty present in the experiment. The apparent current is shown in Figure 10. Current pulses that are qualitatively similar to those observed in this work, i.e. with a magnitude in the range of $\mathrm{mA}$ with a growth time scale in the region of $5 \mathrm{~ns}$ and a decay in the region of 20-30 ns, have been reported in experimental and simulation work in the literature [8, 10, 16, 25]. PDs with these current pulses have been described as 'streamer-like' by Morshuis, [16], and corresponded to the initial stages of discharge activity; the conditions of which this work has sought to replicate.

\begin{tabular}{cc} 
Applied Voltage (kV) & Apparent Charge (pC) \\
\hline \hline 9 & 176 \\
9.5 & 187 \\
10 & 204
\end{tabular}

Table 3: Apparent Charge Magnitudes 


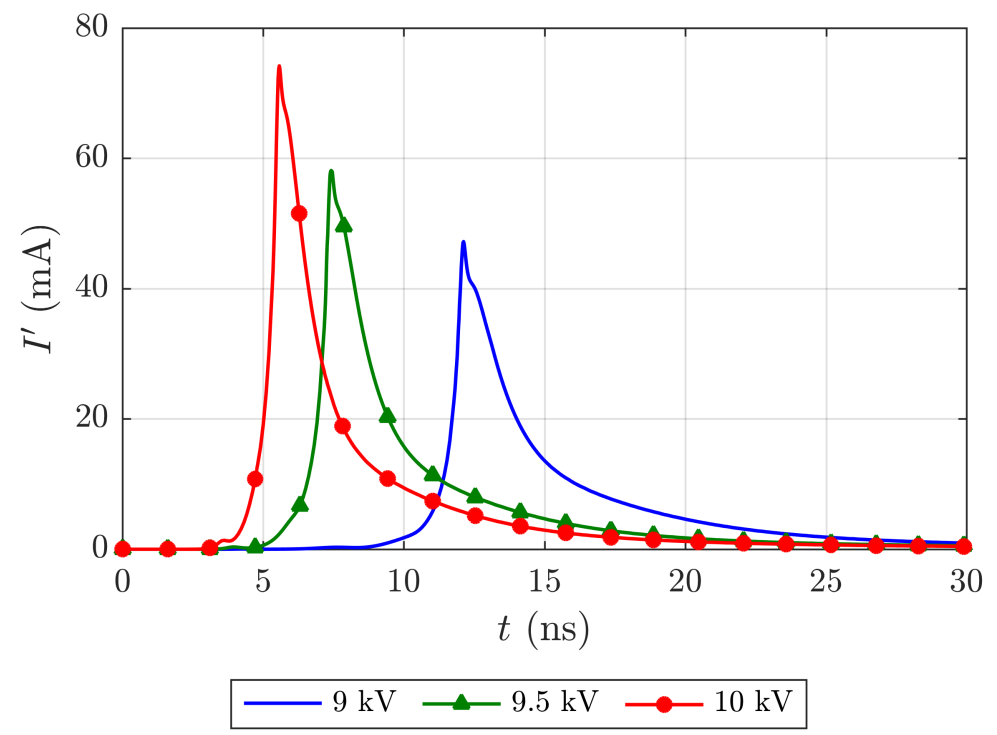

Figure 10: Predicted apparent current on ground electrode at different applied voltages.

In concept 1, see Section 2, an equation for calculating the inception field, i.e. the minimum electric field at which a PD can occur, was introduced. For the conditions present in the system under consideration, the inception field calculated using (1) is $4.7 \mathrm{kV} / \mathrm{mm}$. In the two dimensional model the lowest applied voltage at which a discharge took place was $9 \mathrm{kV}$, which corresponds to an inception field between $5.6 \mathrm{kV} / \mathrm{mm}$ and $5.9 \mathrm{kV} / \mathrm{mm}$ as the applied voltage was increased in increments of $0.5 \mathrm{kV}$. There is therefore reasonable agreement with the inception field formula (1). A noticeable disagreement with canonical reasoning is that the electric field drops to a residual value after the discharge. For the system under consideration, this residual value is $0.88 \mathrm{kV} / \mathrm{mm}$, calculated using (2). Instead of falling to this value, the electric field inside the void is significantly lower immediately after the discharge has taken place, see Figure 11, except in regions close to the top of the void, which are also shorted out, but over longer timescales. 


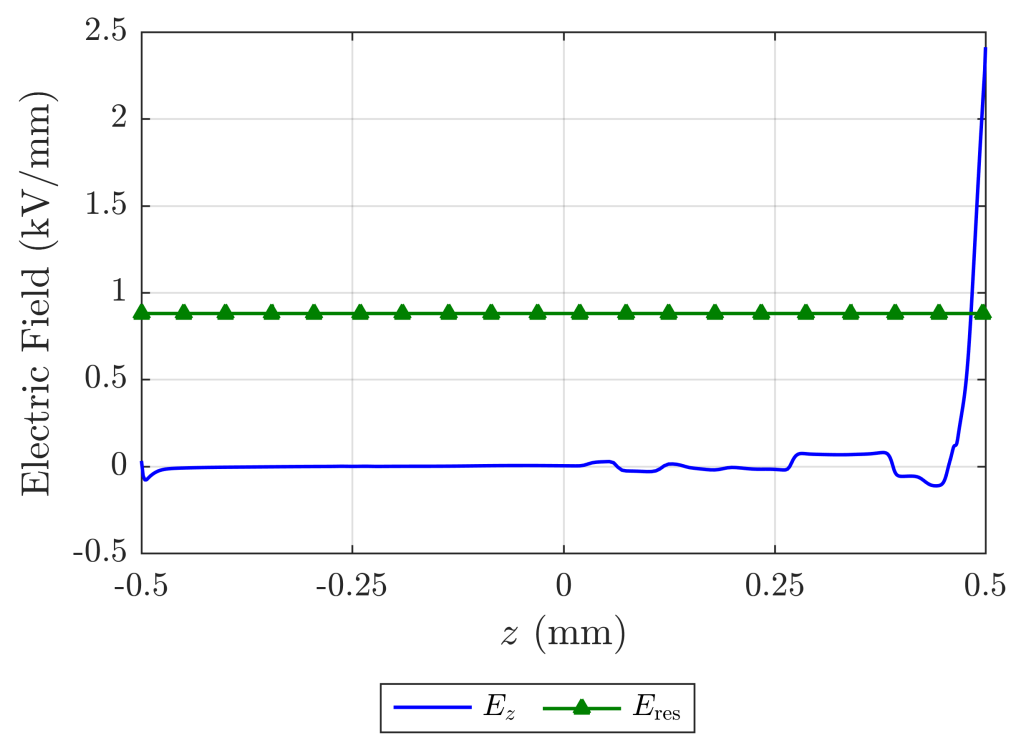

Figure 11: Figure showing the $z$ component of the electric field magnitude the symmetry axis inside the void at $60 \mathrm{~ns}$ for the $9 \mathrm{kV}$ discharge simulation and the residual field calculated using (1).

\subsection{Effect of Subsequent Discharges}

In this configuration the model was initialised with the surface charge density distribution on the void surface set equal to the surface charge density distribution at $-9 \mathrm{kV}$ at $60 \mathrm{~ns}$. As was discussed in the previous section, the field created by this surface charge leads to an electric field in void close to zero. In order to initiate PD an applied voltage of $-18 \mathrm{kV}$ was used, which means the field in the void is roughly equivalent to the electric fields present in the previous section of discharges in virgin voids, with a distortion caused by the surface charge. This neglects the slight change in surface charge that would occur during the time between these discharges due to the remaining plasma cloud, but it is not thought that this would significantly influence the presented results.

The physics of the discharge are similar to the first discharge results, the seed charge multiplies and develops into a positive streamer directed towards the the top of the void. For brevity plots of the discharge variables are not shown for a range of times. A noticeable difference to the first discharge results was observed in the distribution of charge in the streamer head due to the electric field caused by the surface charge already present on the void surface. This led to peak number densities and electric fields being away from the symmetry axis in a toroidal distribution. This is caused to the high field region at the void surface due to the gradient in the surface charge density from the previous discharge. The discharge dependent variables during the initial stage of the positive streamer are shown in Figure 12, It should be noted the toroidal distributions may be due to the use of an axisymmetric model instead of a fully three dimensional model. However, the use of an axisymmetric model is sufficient to explore whether surface charge density from previous PDs is deposited on top of previous PDs, and if the dynamics of the discharge are significantly different in subsequent discharges, which is the purpose of this investigation. If a fully three dimensional model was used it is expected that the plasma dynamics would be that of a positive streamer propagating 'off axis.' 


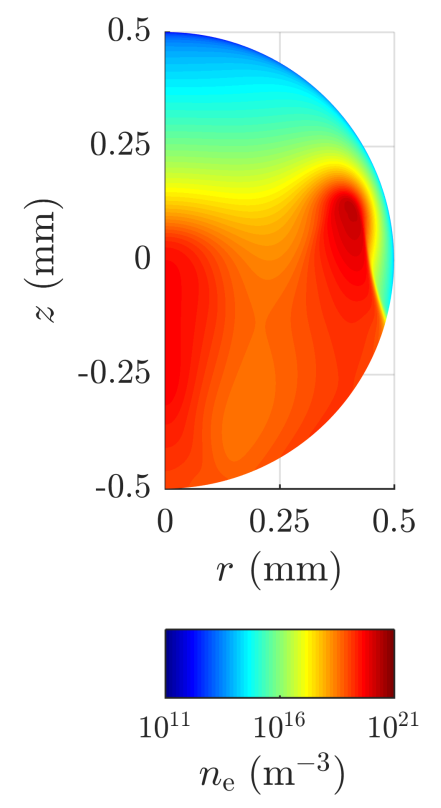

(a)

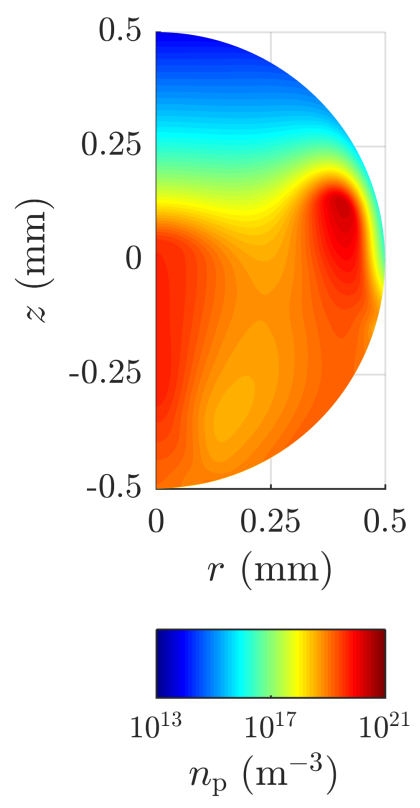

(b)
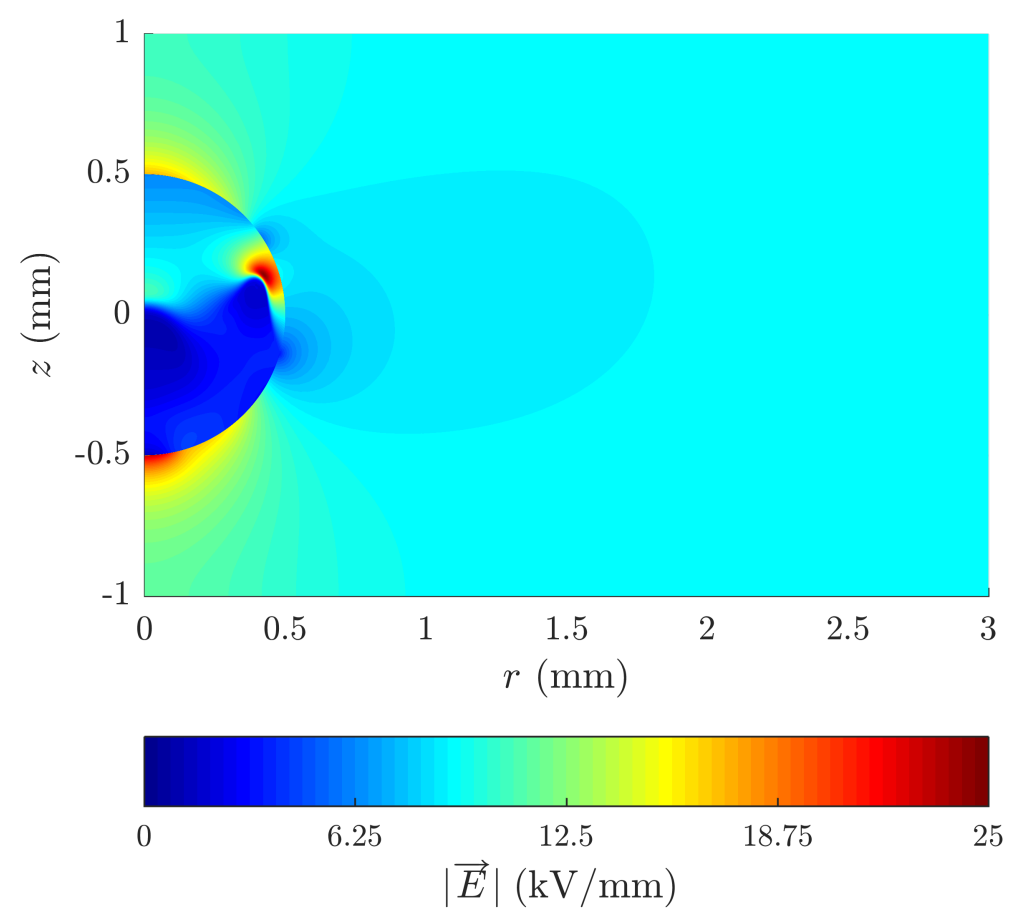

(c)

Figure 12: Discharge dependent variables at 6 ns for the subsequent discharge: (a) electron number density, (b) positive ion number density, (c) electric field magnitude.

The surface charge deployed by the subsequent discharge has a wider distribution, and it did not sit directly 'on top' of the surface charge already at the void boundary, see Figure 13 as is currently assumed 
in PD activity models [6]. This is because the surface charge from the discharge at $-9 \mathrm{kV}$ repelled the charge deposited by the subsequent discharge at $-18 \mathrm{kV}$, which led to a peak surface charge density away from the symmetry axis in the subsequent discharge. The resulting charge distribution was closer to bipolar than the distribution from a single discharge. Measurable quantities for the discharge were similar to the results for virgin voids, with an apparent charge magnitude of $220 \mathrm{pC}$ and an apparent current pulse with a similar shape with a peak current of $100 \mathrm{~mA}$. This is an important result, because although the surface charge density distributions and discharge dynamics were noticeable different, there were not significant differences in the measurable quantities of the discharge. This implies that it is not possible to tell that the surface charge density distributions are significantly different by the apparent charge and apparent current alone.

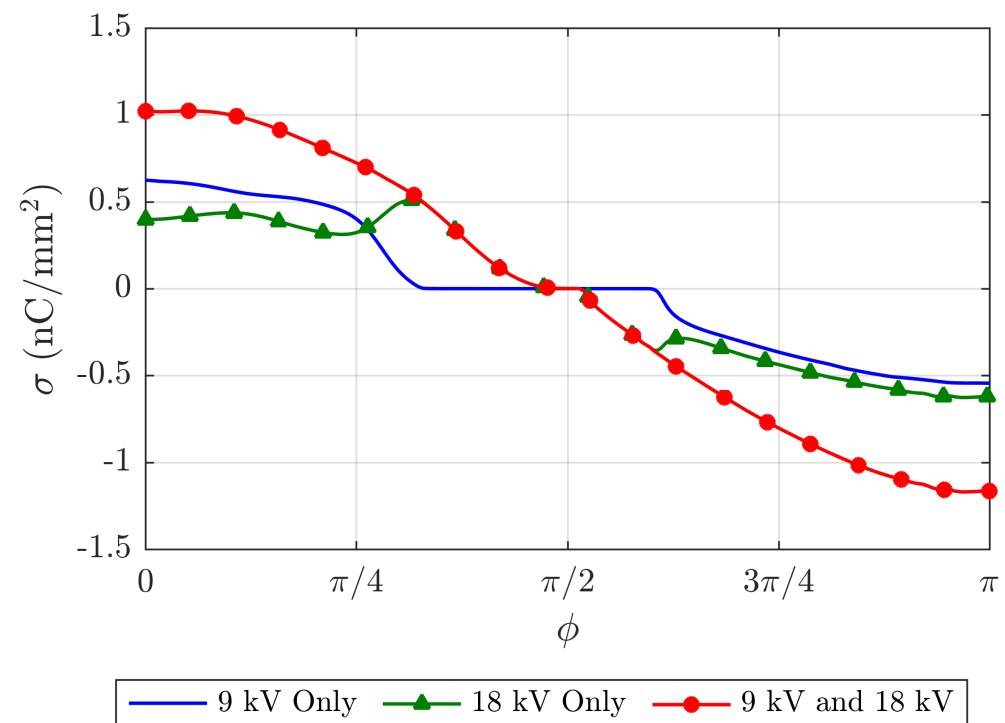

Figure 13: Polar plot of surface charge density on the void surface due to the discharge at $9 \mathrm{kV}$, the subsequent discharge at $18 \mathrm{kV}$ and the resulting distribution.

This final configuration investigated the impact of subsequent discharges. The results were broadly as expected, with the physics of the second discharge comparable to the discharges in virgin voids. However, the charge from the second discharge was not deposited 'on top' of the charge from earlier discharges Instead the charge from the second discharge was more widely distributed due to the electric field from the charge already on the surface opposing its deployment. An interesting point is that although the surface charge density deposited by the second PD was significantly different to the first, the quantities that could be measured experimentally, apparent current and apparent charge, were not significantly different. Previous work in the literature has considered the influence of surface charge density on single PDs, [38, but to the authors knowledge the surface charge density initially was assumed to have a Gaussian distribution, whereas in this work the surface charge density was set based on the distribution of an earlier discharge.

\section{Conclusions}

A drift diffusion model has been implemented to investigate PDs inside a spherical air filled void in conditions which are indicative of the initial stages of discharge activity, which could last in the order of a thousand $50 \mathrm{~Hz}$ AC cycles. The results from the model display a good agreement to measurements of surface charge density distributions, apparent current pulse shapes and apparent charge magnitudes, and previous numerical simulations [8, 16, 25. Nevertheless, it should be noted that the model is relatively simplistic compared to a full model containing all plasma chemistry and boundary phenomena, [11, 12, 32, but in spite of this the 
results provide a test of some of the concepts employed when modelling PD activity. The key contributions of this work as it pertains to PD activity modelling are as follows:

- The surface charge density from PD in spherical voids is not bipolar, a result that has been observed in experimental work [25] and previous simulations for cylindrical voids 8 .

- The inception field predicted by the discharge model was in reasonable agreement with the formula from the literature. It is advisable that the origins of the inception field equation should be considered when using it to model PD activity.

- After the discharge the electric field in the void is significantly reduced, to around $0.1 \mathrm{kV} / \mathrm{mm}$, and is far lower than the residual field predicted by earlier work [5]. As such the assumption that a residual field exists at the order of $1 \mathrm{kV} / \mathrm{mm}$ may be erroneous for PD under certain conditions.

The key contributions of this work as it pertains to plasma dynamics are as follows:

- The boundary conditions for the Helmholtz equations, used to calculate the photoionisation rate, can be significantly simplified for the system under consideration to vastly reduce their computational cost.

- A drift diffusion plasma model with swarm parameters was able to reproduce the measurable quantities of $\mathrm{PD}$, namely apparent current and charge.

- The plasma dynamics of PD in spherical air filled voids, at the conditions considered, consist of an electron avalanche transitioning into a positive streamer. This is followed by charge deposition at the void boundary. These dynamics are in agreement with more complex simulation work [12, 13.

- A subsequent discharge was found to have similar dynamics to the discharges in virgin voids, with comparable measurable quantities. However, the peak charged species number densities and electric fields of the discharge were reached 'off axis' due to the enhancement of the electric field from the surface charge on the void surface from the previous discharge.

\section{Acknowledgments}

The authors acknowledge the use of the IRIDIS High Performance Computing Facility and associated support services at the University of Southampton. This work was supported by an EPSRC Doctoral Training Centre grant $(\mathrm{EP} / \mathrm{G} 03690 \mathrm{X} / 1)$.

\section{References}

[1] R.J. Van Brunt. Physics and chemistry of partial discharge and corona. Recent advances and future challenges. Dielectrics and Electrical Insulation, IEEE Transactions on, 1(5):761-784, 1994.

[2] R. Bartnikas. Partial discharges their mechanism, detection and measurement. Dielectrics and Electrical Insulation, IEEE Transactions on, 9(5):763-808, 2002.

[3] F. Gutfleisch and L. Niemeyer. Measurement and simulation of PD in epoxy voids. Dielectrics and Electrical Insulation, IEEE Transactions on, 2(5):729-743, 1995.

[4] L. Niemeyer, B. Fruth, and F. Gutfleisch. Simulation of partial discharges in insulation systems. In High Voltage Engineering, 1991. 7th International Symposium on, pages 25-28, 1991.

[5] L. Niemeyer. A generalized approach to partial discharge modeling. Dielectrics and Electrical Insulation, IEEE Transactions on, 2(4):510-528, 1995.

[6] H.A. Illias, G. Chen, and P.L. Lewin. The influence of spherical cavity surface charge distribution on the sequence of partial discharge events. Journal of Physics D: Applied Physics, 44(24):245202, 2011.

[7] C. Forssen and H. Edin. Partial discharges in a cavity at variable applied frequency part 2: measurements and modeling. Dielectrics and Electrical Insulation, IEEE Transactions on, 15(6):1610-1616, 2008. 
[8] Y.V. Serdyuk and S.M. Gubanski. Computer modeling of interaction of gas discharge plasma with solid dielectric barriers. Dielectrics and Electrical Insulation, IEEE Transactions on, 12(4):725-735, 2005.

[9] L. Testa, S. Serra, and G.C. Montanari. Advanced modeling of electron avalanche process in polymeric dielectric voids: Simulations and experimental validation. Journal of Applied Physics, 108(3):034110, 2010.

[10] C. Pan, Y. Meng, K. Wu, Z. Han, K. Qin, and Y. Cheng. Simulation of partial discharge sequences using fluid equations. Journal of Physics D: Applied Physics, 44(25):255201, 2011.

[11] A. Villa, L. Barbieri, M. Gondola, A.R. Leon-Garzon, and R. Malgesini. A pde-based partial discharge simulator. Journal of Computational Physics, 345:687-705, 2017.

[12] N.Y. Babaeva, D.V. Tereshonok, and G.V. Naidis. Initiation of breakdown in bubbles immersed in liquids: pre-existed charges versus bubble size. Journal of Physics D: Applied Physics, 48(35):355201, 2015.

[13] N.Y. Babaeva, G.V. Naidis, D.V. Tereshonok, and B.M. Smirnov. Streamer breakdown in elongated, compressed and tilted bubbles immersed in water. Journal of Physics D: Applied Physics, 50(36):364001, 2017.

[14] N.Y. Babaeva and M.J. Kushner. Structure of positive streamers inside gaseous bubbles immersed in liquids. Journal of Physics D: Applied Physics, 42(13):132003, 2009.

[15] H.A. Illias, G. Chen, and P.L. Lewin. Partial discharge behavior within a spherical cavity in a solid dielectric material as a function of frequency and amplitude of the applied voltage. Dielectrics and Electrical Insulation, IEEE Transactions on, 18(2):432-443, 2011.

[16] P.H.F Morshuis. Partial discharge mechanisms. PhD thesis, Delft University of Technology, 1993.

[17] P. Dordizadeh, K. Adamiak, and G.S. Peter Castle. Study of the impact of photoionization on negative and positive needle-plane corona discharge in atmospheric air. Plasma Sources Science and Technology, 25(6):065009, 2016.

[18] T.N. Tran, I.O. Golosnoy, P.L. Lewin, and G.E. Georghiou. Numerical modelling of negative discharges in air with experimental validation. Journal of Physics D: Applied Physics, 44(1):015203, 2011.

[19] D. Braun, V. Gibalov, and G. Pietsch. Two-dimensional modelling of the dielectric barrier discharge in air. Plasma Sources Science and Technology, 1(3):166, 1992.

[20] Y.V. Yurgelenas and M.A. Leeva. Development of a barrier discharge in air in highly nonhomogeneous electric field caused by the residual dielectric surface charges. IEEE Transactions on Plasma Science, 37(6):809-815, 2009.

[21] H.A. Boyd, F.M. Bruce, and D.J. Tedford. Sparkover in long uniform-field gaps. Nature, 210:719-720, 1966.

[22] I. Gallimberti. The mechanism of the long spark formation. Journal de Physique Colloques, 40(7):193-250, 1979.

[23] Partial discharge measurements. IEC 60270, 1998.

[24] G.C. Crichton, P.W. Karlsson, and A. Pedersen. Partial discharges in ellipsoidal and spheroidal voids. Electrical Insulation, IEEE Transactions on, 24(2):335-342, 1989.

[25] K. Wu, C. Pan, Y. Meng, and Y. Cheng. Dynamic behavior of surface charge distribution during partial discharge sequences. Dielectrics and Electrical Insulation, IEEE Transactions on, 20(2):612-619, 2013.

[26] COMSOL Multiphysics Version 5.3 reference manual.

[27] W.S. Kang, J.M. Park, Y. Kim, and S.H. Hong. Numerical study on influences of barrier arrangements on dielectric barrier discharge characteristics. IEEE Transactions on Plasma Science, 31(4):504-510, 2003.

[28] A. Bourdon, V.P. Pasko, N.Y. Liu, S. Célestin, P. Ségur, and E. Marode. Efficient models for photoionization produced by non-thermal gas discharges in air based on radiative transfer and the helmholtz equations. Plasma Sources Science and Technology, 16(3):656, 2007.

[29] G.E. Georghiou, A.P. Papadakis, R. Morrow, and A.C. Metaxas. Numerical modelling of atmospheric pressure gas discharges leading to plasma production. Journal of Physics D: Applied Physics, 38(20):R303, 2005. 
[30] M.B. Zhelezniak, A.K. Mnatsakanian, and S.V. Sizykh. Photoionization of nitrogen and oxygen mixtures by radiation from a gas discharge. High Temperature Science, 20(6):357-362, 1982.

[31] P. Ségur, A. Bourdon, E. Marode, D. Bessieres, and J.H. Paillol. The use of an improved eddington approximation to facilitate the calculation of photoionization in streamer discharges. Plasma Sources Science and Technology, 15(4):648, 2006.

[32] C. Lazarou, T. Belmonte, A.S. Chiper, and G.E. Georghiou. Numerical modelling of the effect of dry air traces in a helium parallel plate dielectric barrier discharge. Plasma Sources Science and Technology, 25(5):055023, 2016.

[33] R.L. Taylor O.C. Zienkiewicz and P. Nithiarasu. The Finite Element Method for Fluid Dynamics, 6th ed. Elsevier, 2005.

[34] R. Codina. Comparison of some finite element methods for solving the diffusion-convection-reaction equation. Computer Methods in Applied Mechanics and Engineering, 156(1):185-210, 1998.

[35] G. Hauke and T.J.R. Hughes. A comparative study of different sets of variables for solving compressible and incompressible flows. Computer Methods in Applied Mechanics and Engineering, 153(1):1-44, 1998.

[36] P.H.F. Morshuis. Partial discharge mechanisms in voids related to dielectric degradation. Science, Measurement and Technology, IEE Proceedings, 142(1):62-68, 1995.

[37] G. Callender, P. Rapisarda, and P.L. Lewin. Improving models of partial discharge activity using simulation. In Electrical Insulation Conference, 2017. EIC 2017. IEEE, June 2017.

[38] V. Nikonov, R. Bartnikas, and M.R. Wertheimer. The influence of dielectric surface charge distribution upon the partial discharge behavior in short air gaps. IEEE Transactions on Plasma Science, 29(6):866-874, 2001. 